\title{
Refinement of a Previous Hypothesis of the Lyapunov Analysis of Isotropic Turbulence
}

\author{
Nicola de Divitiis \\ Dipartimento di Ingegneria Meccanica e Aerospaziale, "La Sapienza” University, Via Eudossiana 18, 00184 Rome, Italy \\ Correspondence should be addressed to Nicola de Divitiis; n.dedivitiis@gmail.com
}

Received 18 August 2012; Accepted 15 March 2013

Academic Editor: Mickaël Lallart

Copyright (C) 2013 Nicola de Divitiis. This is an open access article distributed under the Creative Commons Attribution License, which permits unrestricted use, distribution, and reproduction in any medium, provided the original work is properly cited.

\begin{abstract}
The purpose of this paper is to improve a hypothesis of the previous work of N. de Divitiis (2011) dealing with the finite-scale Lyapunov analysis of isotropic turbulence. There, the analytical expression of the structure function of the longitudinal velocity difference $\Delta u_{r}$ is derived through a statistical analysis of the Fourier transformed Navier-Stokes equations and by means of considerations regarding the scales of the velocity fluctuations, which arise from the Kolmogorov theory. Due to these latter considerations, this Lyapunov analysis seems to need some of the results of the Kolmogorov theory. This work proposes a more rigorous demonstration which leads to the same structure function, without using the Kolmogorov scale. This proof assumes that pair and triple longitudinal correlations are sufficient to determine the statistics of $\Delta u_{r}$ and adopts a reasonable canonical decomposition of the velocity difference in terms of proper stochastic variables which are adequate to describe the mechanism of kinetic energy cascade.
\end{abstract}

\section{Introduction}

In the previous work [1], the author applies the finitescale Lyapunov theory to analyse the homogeneous isotropic turbulence. In particular, this theory leads to the analytical closure of the von Kármán-Howarth equation, giving the longitudinal triple velocity correlation $k$ in terms of the longitudinal velocity correlation $f$ and $\partial f / \partial r$ (see also the appendix), and shows that the structure function of the longitudinal velocity difference is expressed by

$$
\frac{\Delta u_{r}}{\sqrt{\left\langle\left(\Delta u_{r}\right)^{2}\right\rangle}}=\frac{\xi+\psi\left(\chi\left(\eta^{2}-1\right)-\left(\zeta^{2}-1\right)\right)}{\sqrt{1+2 \psi^{2}\left(1+\chi^{2}\right)}},
$$

where $\Delta u_{r}=(\mathbf{u}(\mathbf{x}+\mathbf{r})-\mathbf{u}(\mathbf{x})) \cdot \mathbf{r} / r, \xi, \eta$, and $\zeta$ are uncorrelated centered Gaussian random variables, with $\left\langle\xi^{2}\right\rangle=\left\langle\eta^{2}\right\rangle=\left\langle\zeta^{2}\right\rangle$ $=1$, and $\psi$ is a function of the Taylor scale Reynolds number $R_{\lambda}=u \lambda_{T} / \nu$ and of the separation distance $r \equiv|\mathbf{r}|$, according to

$$
\psi\left(r, R_{\lambda}\right)=\sqrt{\frac{R_{\lambda}}{15 \sqrt{15}}} \widehat{\psi}(r),
$$

where $u=\sqrt{\left\langle u_{r}^{2}\right\rangle}$ is the longitudinal velocity standard deviation, $\lambda_{T}$ is the Taylor microscale, and $\chi=\chi\left(R_{\lambda}\right) \neq 1$ is a proper function of $R_{\lambda}$ which provides nonzero skewness of $\Delta u_{r}[1,2]$.

In [1], the demonstration of (1) is carried out through statistical elements regarding the Fourier transformed NavierStokes equations, whereas the proof of (2) is based on the fact that, according to the Kolmogorov theory, the ratio (small scale velocity)-(large scale velocity) depends on $\lambda_{T} / \ell \approx \sqrt{R_{\lambda}}$, where $\ell$ is the Kolmogorov microscale. Therefore, the analysis of [1] seems to require the adoption of $\ell$ whose definition is based on another theory.

Here, instead of using the Kolmogorov scale, we obtain (1) and (2), starting from the canonical decomposition of the fluid velocity in terms of proper centered random variables $\xi_{k}$. In order to describe the mechanism of kinetic energy cascade, the variables $\xi_{k}$ are properly chosen in such a way that each of them exhibits a nonsymmetric distribution function. Moreover, due to the isotropy, we postulate that the knowledge of $f$ and $k$ represents a sufficient condition to determine the statistics of $\Delta u_{r}$. 


\section{The Lyapunov Analysis of the Velocity Fluctuations}

This section renews the procedure for calculating the velocity fluctuations, which is based on the Lyapunov analysis of the fluid strain [1] and on the momentum Navier-Stokes equations

$$
\frac{\partial u_{k}}{\partial t}=-\frac{\partial u_{k}}{\partial x_{h}} u_{h}+\frac{1}{\rho} \frac{\partial T_{k h}}{\partial x_{h}}
$$

where $\mathbf{u} \equiv\left(u_{1}, u_{2}, u_{3}\right), T_{k h}$, and $\rho$ are the fluid velocity, stress tensor, and density, respectively.

In order to obtain the velocity fluctuation, consider now the relative motion between two contiguous particles, expressed by the infinitesimal separation vector $d \mathbf{x}$ which obeys

$$
d \dot{\boldsymbol{x}}=\nabla \mathbf{u} d \mathbf{x}
$$

where $d \mathbf{x}$ varies according to the velocity gradient which in turn follows the Navier-Stokes equations. As observed in [1], $d \mathbf{x}$ is much faster than the fluid state variables, and the Lyapunov analysis of (4) provides the expression of the local deformation in terms of the maximal Lyapunov exponent $\Lambda>0$

$$
\frac{\partial \mathbf{x}}{\partial \mathbf{x}_{0}} \approx e^{\Lambda\left(t-t_{0}\right)}
$$

The map $\chi: \mathbf{x}_{0} \rightarrow \mathbf{x}$ is the function which gives the current position $\mathbf{x}$ of a fluid particle located at the referential position $\mathbf{x}_{0}$ at $t=t_{0}$ [3]. Equation (3) can be written in terms of the referential position $\mathbf{x}_{0}$ [3]

$$
\frac{\partial u_{k}}{\partial t}=\left(-\frac{\partial u_{k}}{\partial x_{0 p}} u_{h}+\frac{1}{\rho} \frac{\partial T_{k h}}{\partial x_{0 p}}\right) \frac{\partial x_{0 p}}{\partial x_{h}} .
$$

The adoption of the referential coordinates allows to factorize the velocity fluctuation and to express it in the Lyapunov exponential form of the local deformation. As this deformation is assumed to be much more rapid than $-\partial u_{k} / \partial x_{0 p} u_{h}+1 / \rho \partial T_{k h} / \partial x_{0 p}$, the velocity fluctuation can be obtained by integrating (6) with respect to the time, where $-\partial u_{k} / \partial x_{0 p} u_{h}+1 / \rho \partial T_{k h} / \partial x_{0 p}$ is considered to be constant

$$
u_{k} \approx \frac{1}{\Lambda}\left(-\frac{\partial u_{k}}{\partial x_{0 p}} u_{h}+\frac{1}{\rho} \frac{\partial T_{k h}}{\partial x_{0 p}}\right)_{t=t_{0}} .
$$

This assumption is justified by the fact that, according to the classical formulation of motion of continuum media [3], the terms into the circular brackets of (6) are considered to be smooth functions of $t$-at least during the period of a fluctuation-whereas the fluid deformation varies very rapidly according to (4)-(5).

\section{Statistical Analysis of Velocity Difference}

As explained in this section, the Lyapunov analysis of the local deformation and some plausible assumptions about the statistics of $\mathbf{u}$ lead to determination of the structure function of $\Delta u_{r}$ and its PDF.

The statistical properties of $\Delta u_{r}$ are here investigated expressing the fluid velocity through the following canonical decomposition [4]:

$$
\mathbf{u}=\sum_{k} \widehat{\mathbf{U}}_{k} \xi_{k}
$$

where $\widehat{\mathbf{U}}_{k}(k=1,2, \ldots)$ are proper coordinate functions of $t$ and $\mathbf{x}$, and $\xi_{k}(k=1,2, \ldots)$ are certain dimensionless independent stochastic variables which satisfy

$$
\begin{gathered}
\left\langle\xi_{k}\right\rangle=0, \quad\left\langle\xi_{i} \xi_{j}\right\rangle=\delta_{i j}, \\
\left\langle\xi_{i} \xi_{j} \xi_{k}\right\rangle=\omega_{i j k} p, \quad|p| \ggg 1,|p| \ggg\left\langle\xi_{k}^{4}\right\rangle,
\end{gathered}
$$

where $\omega_{i j k}=1$ for $i=j=k$, else $\omega_{i j k}=0$. It is worth to remark that the variables $\xi_{k}$ are adequately chosen in such a way that they can describe properly the mechanism of energy cascade. Specifically, the adoption of $\xi_{k}$ with $|p| \ggg 1$ is justified by the fact that the evolution equation of the velocity correlation (see, e.g. the von Kármán-Howarth equation in appendix) includes also the third-order velocity correlation $k(r)$ which is responsible for the intensive mechanism of energy cascade, and $\left\langle\left(\Delta u_{r}\right)^{3}\right\rangle /\left\langle\left(\Delta u_{r}\right)^{2}\right\rangle^{3 / 2} \neq 0$. As a result, it is reasonable that the canonical decomposition (8) $\left(\Delta u_{r}=(\mathbf{r} / r) \cdot \sum_{k}\left(\widehat{\mathbf{U}}_{k}(\mathbf{x}+\mathbf{r})-\right.\right.$ $\left.\left.\widehat{\mathbf{U}}_{k}(\mathbf{x})\right) \xi_{k}\right)$ includes variables $\xi_{k}$ with $\left|\left\langle\xi_{k}^{3}\right\rangle\right| \ggg 1$ [5]. This has very important implications for what concerns the statistics of the fluctiations of $\Delta u_{r}$. In order to analyze this question, consider now the dimensionless velocity fluctuation $\widehat{\mathbf{u}}$. This is obtained in terms of $\xi_{k}$ by substituting (8) into (7)

$$
\widehat{u}_{h}=\sum_{i j} A_{h i j} \xi_{i} \xi_{j}+\frac{1}{R_{\lambda}} \sum_{k} b_{h k} \xi_{k} \text {, }
$$

where $r=\widehat{r} \lambda_{T}$ and $u_{h}=\widehat{u}_{h} u$. Therefore $\sum_{i j} A_{h i j} \xi_{i} \xi_{j}$ arises from the inertia and pressure terms, whereas $1 / R_{\lambda} \sum_{k} b_{h k} \xi_{k}$ is due to the fluid viscosity. Now, thanks to the local isotropy, $u_{h}$ is a Gaussian stochastic variable $[4,5]$; accordingly, $\xi_{k}$ satisfy into (10), the Lindeberg condition, a very general, necessary, and sufficient condition for satisfying the central limit theorem [5]. This condition does not apply to the velocity difference. In fact, as $\Delta \mathbf{u}$ is the difference between two correlated Gaussian variables, its PDF could be a nonGaussian distribution function. To study this, the fluctuation $\Delta u_{r}$ is first expressed in terms of $\xi_{k}$

$$
\begin{aligned}
\Delta \widehat{u}_{r}(\mathbf{r}) & =\sum_{i j} \Delta A_{r i j}(\mathbf{r}) \xi_{i} \xi_{j}+\frac{1}{R_{\lambda}} \sum_{k} \Delta b_{r k}(\mathbf{r}) \xi_{k} \\
& \equiv L+S+G^{+}+G^{-} .
\end{aligned}
$$

This fluctuation can be reduced to the contributions $L, S$, $G^{+}$, and $G^{-}$, appearing into (11) [6]: in particular, $L$ is the sum of all linear terms due to the fluid viscosity, and $S \equiv S_{i j} \xi_{i} \xi_{j}$ is the sum of all bilinear forms arising from the inertia and pressure terms, whereas $G^{+}$and $G^{-}$are, respectively, definite positive and negative quadratic forms of centered Gaussian 
variables, which are derived from the inertia and pressure terms. The quantity $L+S$ tends to a Gaussian random variable being the sum of statistically orthogonal terms $[5,6]$, while $G^{+}$and $G^{-}$are determined by means of the hypotheses of isotropy and of fully developed flow

$$
\begin{aligned}
G^{-} & =-\left(\zeta^{2}-1\right) \psi_{2}(r), \\
G^{+} & =\left(\eta^{2}-1\right) \psi_{3}(r) .
\end{aligned}
$$

Observe that, due to these hypotheses, $G^{+}$and $G^{-}$ are uncorrelated; thus $\eta, \zeta$ are two independent centered Gaussian variables, with $\left\langle\eta^{2}\right\rangle=\left\langle\zeta^{2}\right\rangle=1$. Furthermore, as the knowledge of $f$ and $k$ is considered to be a sufficient condition for determining the statistics of $\Delta u_{r}, \psi_{2}$ and $\psi_{3}$ are assumed to be proportional with each other through a constant which depends only on $R_{\lambda}$

$$
\chi\left(R_{\lambda}\right)=\frac{\psi_{3}(r)}{\psi_{2}(r)} .
$$

Therefore, the longitudinal velocity difference can be written as

$$
\Delta u_{r}=\psi_{1} \xi+\psi_{2}\left(\chi\left(\eta^{2}-1\right)-\left(\zeta^{2}-1\right)\right),
$$

where $\xi$ is a centered Gaussian random variable with $\left\langle\xi^{2}\right\rangle=1$, that, thanks to the hypotheses of fully developed flow and of isotropy, is considered to be statistically independent from $\eta$ and $\zeta$.

Comparing the terms of (14) and (11), we obtain that $\psi_{1}$ and $\psi_{2}$ are related with each other and that their ratio $\psi \equiv$ $\psi_{1} / \psi_{2}$ depends on $R_{\lambda}$ and $r$

$$
2\left(1+\chi^{2}\right) \frac{\psi_{2}^{2}}{\psi_{1}^{2}}=\frac{\left\langle\left(G^{+}+G^{-}\right)^{2}\right\rangle}{\left\langle\left(S_{i j} \xi_{i} \xi_{j}+\left(1 / R_{\lambda} \Delta b_{r k} \xi_{k}\right)\right)^{2}\right\rangle} .
$$

Now, the divisor at the R. H. S. of (15) is the sum of the following three terms:

$$
\begin{gathered}
A=S_{i j} S_{p q}\left\langle\xi_{i} \xi_{j} \xi_{p} \xi_{q}\right\rangle, \quad B=\frac{2}{R_{\lambda}} S_{i j}\left\langle\xi_{i} \xi_{j} \xi_{k}\right\rangle \Delta b_{r k}, \\
C=\frac{1}{R_{\lambda}^{2}} \Delta b_{r k}^{2}\left\langle\xi_{k}^{2}\right\rangle .
\end{gathered}
$$

Hence, taking into account the properties (9) of $\xi_{k},|B| \gg$ $|A|,|C|$; thus $\psi$ tends to a quantity arising only from the terms $\left\langle\xi_{k}^{3}\right\rangle$ which appear in (15):

$$
\psi \equiv \frac{\psi_{2}}{\psi_{1}}=\varphi(r) \sqrt{R_{\lambda}}
$$

This expression corresponds to that obtained in [1]

$$
\psi\left(r, R_{\lambda}\right)=\sqrt{\frac{R_{\lambda}}{15 \sqrt{15}}} \widehat{\psi}(r), \quad \widehat{\psi}(0)=O(1),
$$

and the dimensionless longitudinal velocity difference is given by (1)

$$
\frac{\Delta u_{r}}{\sqrt{\left\langle\left(\Delta u_{r}\right)^{2}\right\rangle}}=\frac{\xi+\psi\left(\chi\left(\eta^{2}-1\right)-\left(\zeta^{2}-1\right)\right)}{\sqrt{1+2 \psi^{2}\left(1+\chi^{2}\right)}} .
$$

It is worth to remark that $\psi$ expresses the fluctuations ratio (large scale velocity)-(small scale velocity); that is, $\psi \approx$ $u / u_{s} \approx\left(u^{2} / \lambda_{T}\right)\left(l_{s} / u_{s}^{2}\right)$, where $l_{s}$ and $u_{s}$ are, respectively the characteristic small scale and the corresponding velocity. This implies that $u / u_{s} \simeq \lambda_{T} / l_{s} \approx \sqrt{R_{\lambda}}$; thus $l_{s}$ identifies the Kolmogorov scale, and $u_{s} l_{s} / \nu \approx 1$ is the corresponding Reynolds number.

The distribution function of $\Delta u_{r}$ is then expressed through the Frobenius-Perron equation [7], taking into account that $\xi, \eta$, and $\zeta$ are independent stochastic variables

$$
\begin{aligned}
& F\left(\Delta u_{r}^{\prime}\right) \\
& \quad=\iint_{\xi} \int_{\eta} p(\xi) p(\eta) p(\zeta) \delta\left(\Delta u_{r}^{\prime}-\Delta u_{r}(\xi, \eta, \zeta)\right) d \xi d \eta d \zeta,
\end{aligned}
$$

where $\Delta u_{r}(\xi, \eta, \zeta)$ is determined by (19), $\delta$ is the Dirac delta, and $p$ is a centered Gaussian PDF with standard deviation equal to the unity. The dimensionless statistical moments of $\Delta u_{r}$ are easily calculated

$$
\begin{aligned}
H_{n} \equiv & \frac{\left\langle\left(\Delta u_{r}\right)^{n}\right\rangle}{\left\langle\left(\Delta u_{r}\right)^{2}\right\rangle^{n / 2}} \\
= & \frac{1}{\left(1+2\left(1+\chi^{2}\right) \psi^{2}\right)^{n / 2}} \\
& \times \sum_{k=0}^{n}\left(\begin{array}{l}
n \\
k
\end{array}\right) \psi^{k}\left\langle\xi^{n-k}\right\rangle\left\langle\left(\chi\left(\eta^{2}-1\right)-\left(\zeta^{2}-1\right)\right)^{k}\right\rangle .
\end{aligned}
$$

In particular, $\mathrm{H}_{3}$, related to the mechanism of energy cascade, is

$$
H_{3}(r)=\frac{8 \psi^{3}\left(\chi^{3}-1\right)}{\left(1+2 \psi^{2}\left(1+\chi^{2}\right)\right)^{3 / 2}} .
$$

In conclusion, $\chi=\chi\left(R_{\lambda}\right)$ is implicitly calculated, in function of $\psi(0)$, taking into account that this Lyapunov theory gives $H_{3}(0)=-3 / 7$ (see appendix) [1]

$$
H_{3}(0)=\frac{8 \psi^{3}(0)\left(\chi^{3}-1\right)}{\left(1+2 \psi^{2}(0)\left(1+\chi^{2}\right)\right)^{3 / 2}}=-\frac{3}{7},
$$

where $\widehat{\psi}(0) \simeq 1.075$ is estimated as in $[1]$, and $\sqrt{\left\langle\left(\Delta u_{r}\right)^{2}\right\rangle}$ and $\widehat{\psi}(r)$ are calculated in function of $f(r)$ and $k(r)$.

We conclude this paper by observing that the mechanism of energy cascade acts on $\Delta u_{r}$ whose expression, here calculated with the finite-scale Lyapunov theory and (9), provides a nonsymmetric PDF, where the absolute values of the dimensionless moments $\left|H_{n}(0)\right|$ rise with the Taylor scale Reynolds number for $n>3$. 


\section{Appendix}

For the sake of convenience, this section reports some of the results dealing with the closure of the von Kármán-Howarth equation, obtained in $[1,2]$.

For fully developed isotropic homogeneous turbulence, the pair correlation function

$$
f(r)=\frac{\left\langle u_{r}(\mathbf{x}) u_{r}(\mathbf{x}+\mathbf{r})\right\rangle}{u^{2}}
$$

satisfies the von Kármán-Howarth equation [8]

$$
\frac{\partial f}{\partial t}=\frac{K(r)}{u^{2}}+2 v\left(\frac{\partial^{2} f}{\partial r^{2}}+\frac{4}{r} \frac{\partial f}{\partial r}\right)+\frac{10 \nu}{\lambda_{T}^{2}} f
$$

the boundary conditions of which are

$$
f(0)=1, \quad \lim _{r \rightarrow \infty} f(r)=0,
$$

where $u \equiv \sqrt{\left\langle u_{r}^{2}(\mathbf{x})\right\rangle}$ satisfies the equation of the turbulent kinetic energy [8]

$$
\frac{d u^{2}}{d t}=-\frac{10 v}{\lambda_{T}^{2}} u^{2}
$$

and $\lambda_{T} \equiv \sqrt{-1 / f^{\prime \prime}(0)}$ is the Taylor scale. The function $K(r)$, giving the mechanism of energy cascade, is related to the longitudinal triple velocity correlation function $k$

$$
\begin{gathered}
K(r)=u^{3}\left(\frac{\partial}{\partial r}+\frac{4}{r}\right) k(r), \\
\text { where } k(r)=\frac{\left\langle u_{r}^{2}(\mathbf{x}) u_{r}(\mathbf{x}+\mathbf{r})\right\rangle}{u^{3}} .
\end{gathered}
$$

Thus, the von Kármán-Howarth equation provides the relationship between the statistical moments $\left\langle\left(\Delta u_{r}\right)^{2}\right\rangle$ and $\left\langle\left(\Delta u_{r}\right)^{3}\right\rangle$ in function of $r$.

The Lyapunov theory proposed in [1] gives the closure of the von Kármán-Howarth equation and expresses $K(r)$ in terms of $f$ and $\partial f / \partial r$

$$
K(r)=u^{3} \sqrt{\frac{1-f}{2}} \frac{\partial f}{\partial r} .
$$

Accordingly, the skewness of $\Delta u_{r}$ is [9]

$$
H_{3}(r) \equiv \frac{\left\langle\left(\Delta u_{r}\right)^{3}\right\rangle}{\left\langle\left(\Delta u_{r}\right)^{2}\right\rangle^{3 / 2}}=\frac{6 k(r)}{(2(1-f(r)))^{3 / 2}} .
$$

Therefore, the skewness of $\partial u_{r} / \partial r$ is

$$
H_{3}(0)=-\frac{3}{7}
$$

\section{Acknowledgments}

This work was partially supported by the Italian Ministry for the Universities and Scientific and Technological Research (MIUR).

\section{References}

[1] N. de Divitiis, "Lyapunov analysis for fully developed homogeneous isotropic turbulence," Theoretical and Computational Fluid Dynamics, vol. 25, no. 6, pp. 421-445, 2011.

[2] N. de Divitiis, "Self-similarity in fully developed homogeneous isotropic turbulence using the lyapunov analysis," Theoretical and Computational Fluid Dynamics, vol. 26, no. 1-4, pp. 81-92, 2012.

[3] C. Truesdell, A First Course in Rational Continuum Mechanics, Academic Press, New York, NY, USA, 1977.

[4] E. S. Ventsel, Theorie Des Probabilites, Editions Mir, CCCP, Moskow, 1973.

[5] E. L. Lehmann, Elements of Large-Sample Theory, Springer, 1999.

[6] W. G. Madow, "Limiting distributions of quadratic and bilinear forms," The Annals of Mathematical Statistics, vol. 11, no. 2, pp. 125-146, 1940.

[7] G. Nicolis, Introduction to Nonlinear Science, Cambridge University Press, 1995.

[8] T. de Kármán and L. Howarth, "On the statistical theory of isotropic turbulence," Proceedings of the Royal Society A, vol. 164, no. 917, pp. 192-215, 1938.

[9] G. K. Batchelor, The Theory of Homogeneous Turbulence, Cambridge University Press, Cambridge, Mass, USA, 1953. 

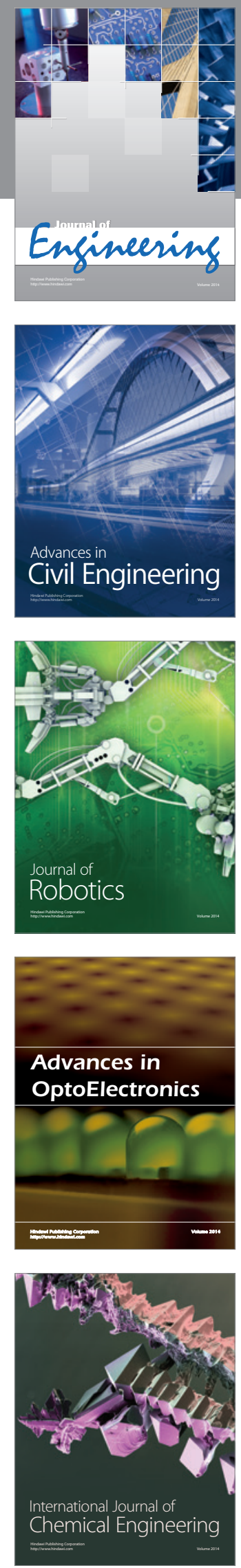

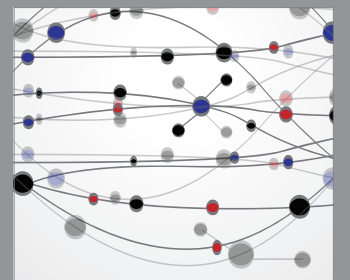

The Scientific World Journal
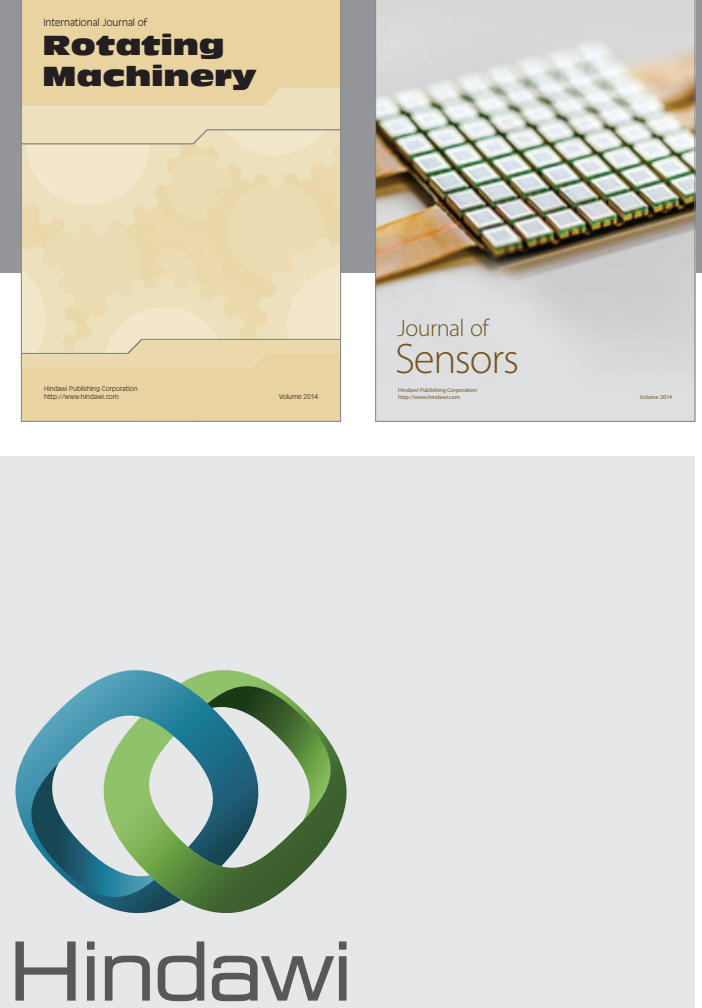

Submit your manuscripts at http://www.hindawi.com
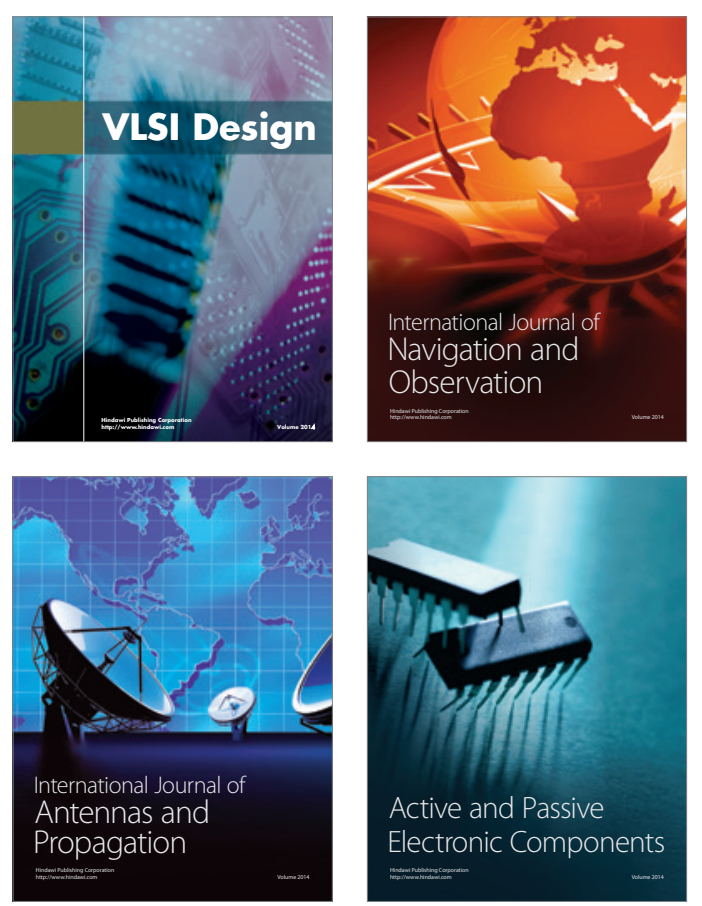
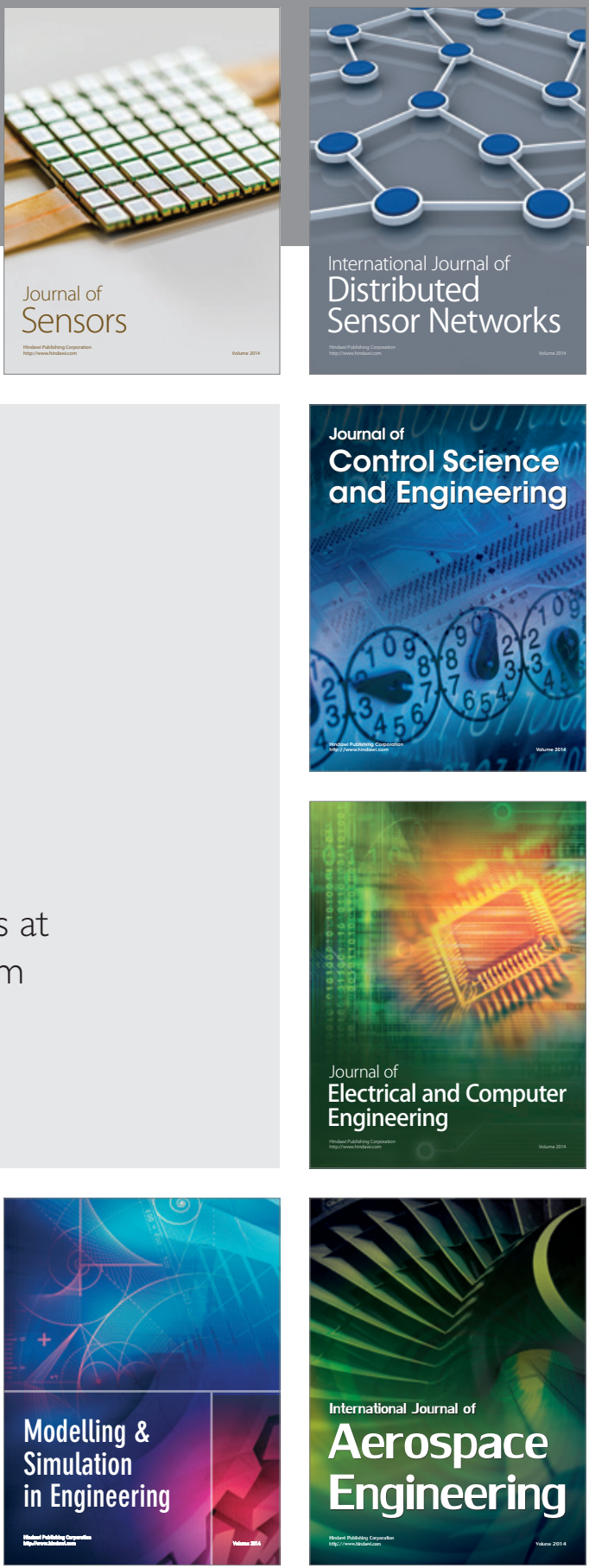

Journal of

Control Science

and Engineering
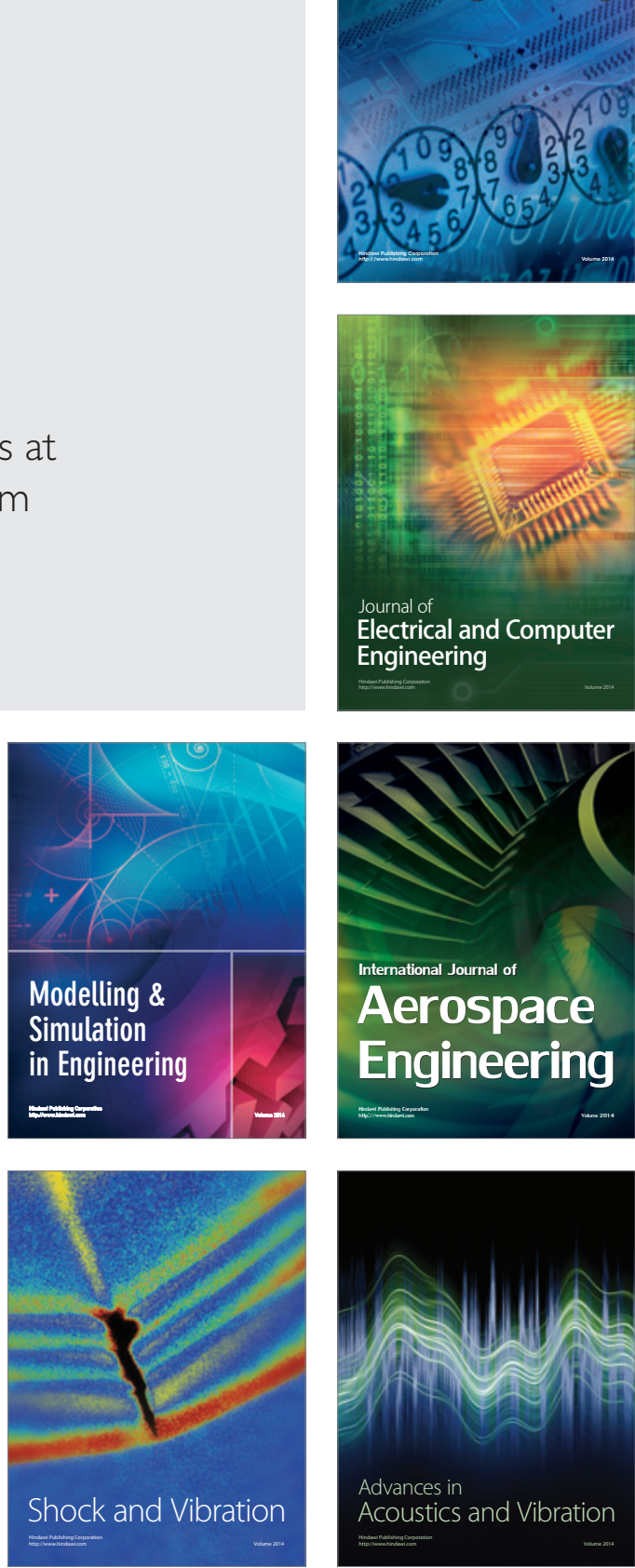